struction with the care of the sick in a free dispensary is important from many standpoints. Its advantages for the student and physician are self evident. Its advantages for the patient are, however, not always understood by the public. They are, nevertheless, very great - as great in the out-patient department as in the wards of the hospital. The presence of students of medicine, undergraduates, or post-graduates, is the greatest stimulus to the careful investigation of the individual case, and to study and research in general. The student as an assistant in the wards and in the out-patient department of a hospital is absolutely invaluable. No hospital which closes its wards to students can ever hope to compete in the quality of its work or in the care given to the patients with institutions in which ward instruction is given. I have known and served in hospitals of both classes, and I know whereof I speak. The same is true to even a greater extent in out-patient departments.

There is one point, however, which has not sufficiently been emphasized, and that is the therapeutic value to the patient himself of medical instruction. I rarely make a ward visit with the students or give a public clinic without the consciousness that what I am saying to the class is of material value to many of the patients. It is almost invariably true that advice given in public and explained at the same time to the student has considerably more force than when it is given directly to the patient. The suspicious, nervous invalid rarely doubts the sincerity of advice given thus in the presence of others, and many a time $I$ have seen the patient induced to the urgent operation only after hearing the pros and cons considerately discussed before the class.

Especially valuable, often, is the combination of instruction with treatment of patients effected with various nervous diseases. One of the most fascinating and inspiring clinies that $I$ know is the out-patient consulting hour of Professor Dejerine at the Salpetrière in Paris. Dejerine here combines his questions and advice to the patient with explanations, confidences and digressions directed more particularly to his audience, in a manner so informal and so sympathetic that one feels immediately that the significance of his words to the patients is appreciably greater than if the advice were given in private. And it is interesting to see how true a bond of sympathy' is formed between these sufferers and the earnest men in the audience, with whom, perhaps, not a word is exchanged. The patient is interested in the fact that others are interested in her case. She is cheered, not only by the helpful words of the doctor, but by the sympathetic glances of the onlookers, and she is reassured by the remarks and comments made to this audience, in a confidential and intimate manner, in the discussion of her case. No one can doubt that in such a clinic as this the combination of instruction with treatment is beneficial to all concerned.
The prime essentials for an efficient dispensary are that it be a part of, or intimately associated with, an institution of learning, and that it should possess a proper equipment for the study and treatment of disease. It is with peculiar satisfaction that I learn that one floor of this building, the cornerstone of which has been laid today, is to be devoted to laboratories. Twenty-five years ago, what laboratory work was necessary in a dispensary, could be done by the clinical assistants themselves. Today, the story is very different, and it is difficult to imagine a dispensary, not associated with an institution of learning, which could afford the necessary equipment or guarantee a staff of a character suitable to carry on the scientific work of the institution:

What a wonderful field of study it is-the study of disease, offering as it does today, problems which demand excursions into all of the natural sciences, problems which offer to the mind of the student the fascination and charm which are attached to any scientific investigation, quickened by the added consciousness of the ends in view, the relief of human suffering, the increase of human efficiency, the prolongation of human life, the betterment of man!

Every hospital which opens its doors to the student of medicine, every university which gives of its resources material and moral, for the establishment of institutions such as that of which today you are laying the cornerstone, is contributing to these great ends.

Of the specific value of a well equipped university dispensary to the public, to the student, to the physician, to the community, I have already spoken, and coming from an institution which stands sorely in need of a building just such as that which you are now erecting, I offer you, not without a touch of, may I say, friendly envy, my cordial congratulations.

\section{(Briginal Artirlpr.}

\section{THE CLOSURE OF OBSTINATE PERIN-} EAL FISTULAE FOLLOWING OPERATION FOR STRICTURE OF URETHRA. THE PREVENTION OF THESE FISTULAE.

BY HOWARD A. LOTHROP, A.M., M.D., BOSTON,

Assistant Professor of Suryery, Harvard Medical School; Visiting Surgeon, Boston City Hospital.

As a general rule, it is essential to discover and remove the cause in treating lesions of a surgical nature, and unless that is done the effort will fail. This is particularly true of cases of obstinate perineal fistulae secondary to lesions of the prostate gland and urethra. Except in the rare cases where tuberculosis and carcinoma are present, the persisting cause is a mechanical one. Like any fluid, urine will follow the course 
of least resistance. Therefore, if there exists a urethra with incomplete wall, accompanied by more or less distal obstruction, one cannot expect the urine to follow the natural channel. Hence, many perineal wounds are either very slow to heal or may remain open indefinitely.

In trying to ascertain the causes of these obstinate and troublesome fistulae, let us consider some of the types of lesions which are followed by this complication.

Group 1. Cases of Urinary Extravasation. These are generally the result of stricture. There is a varying degree of local infiltration, the parts become infected, and the less resistant tissues become necrotic. Meanwhile, the patient may become very toxic and his condition critical unless incisions are made or openings in the skin appear spontaneously. If resolution should follow, a fistula would result and persist indefinitely, because the underlying cause (stricture) still persists. If, however, such a case of extravasation were operated upon simply by free incisions and nothing further done to relieve the stricture, owing perhaps to the serious toxic condition, there would be the same chance of a chronic fistula as though the opening were spontaneous.

It frequently happens in this class of cases, owing to the distortion of parts and the sloughing, that no guide can be passed into the bladder and that in the effort to find the urethra it is extensively injured and perhaps destroyed for some distance or a considerable portion of it sidetracked by the catheter, if passed through the penile urethra into the bladder. Finally, the catheter may have been inserted into the bladder only through the perineum and prostatic urethra. These circumstances tend to establish obstinate perineal drainage.

In dealing with these cases of urinary extravasation, the first indication is the establishment of free drainage from the tissues, particularly if the condition is critical, but, ultimately, of course, the stricture, must be incised. This should be done at the first operation if the condition of the patient warrants, provided the operation is not too prolonged and the effort not attended with too great difficulty or unnecessary injury to the urethra. The bladder should be drained in these cases, as in all others, by a catheter which includes the whole urethra, and the opening in the perineum should serve to drain only the adjacent tissues.

Group 2. Fistulae after Prostatectomy by the Perineal Route. After prostatectomy by the perineal route, chronic and sometimes permanent fistulae are not uncommon. In view of the frequent extensive destruction of the membranous and prostatic urethra and the neglect to keep open the remaining portion of the anterior urethra, it is surprising that the urine finds its way into the penile urethra as well as it does. In some cases careful operators are able to remove the gland without material injury to the urethra but, as a rule, it is considerably torn.
Many resort solely to perineal drainage and occasionally insert considerable packing into the wound and neglect the distal portion of the urethra which, of course, remains collapsed. After these drains are removed, the greater portion of the urine will escape through the wound, but will decrease gradually as the wound tends to close and the distal opening in the urethra remains patent. If granulations tend to obstruct the latter they offer resistance, and a fistula may persist.

If, on the other hand, the operation be performed with care so as to cause a minimum of urethral injury, and a catheter for bladder drainage be placed so as to include the whole urethra, and a light gauze packing be left in the wound and removed as soon as hemorrhage is arrested, then the conditions are most favorable for early and permanent wound closure. The soft parts close around the catheter, where the urethra may be deficient, and the approximated surfaces become glued together by granulation tissue. In many cases the wound may be nearly closed with large, mass, silkworm gut sutures and, by means of seepage for 36 to 48 hours, all exudate and fluids be promptly removed and thereby absorption prevented. The patient may be propped up in bed during this period. If there is no contra-indication, the catheter should remain in place at least one week.

Group 3. Fistulae Following Operation for Stricture. This complication not infrequently follows external urethrotomy, depending upon various circumstances. If we have to deal with a passable stricture, it is possible to obtain relief by intra-urethral methods, such as gradual dilation or internal urethrotomy, irrespective of whether the stricture is situated in the penile or membranous urethra. Internal urethrotomy is the accepted method of treating penile strictures, but is generally condemned for deep strictures because of the danger of infecting the perineal tissues, and this complication has occasionally proved serious. As a rule, however, internal treatment of the urethra is not followed by urinary extravasation or sepsis, even when the crude and now discarded method of divulsion of deep strictures was used. The usual method of treating deep passable strictures which do not yield to gradual dilatation is by external urethrotomy, and this is the only way if no guide can be passed. The resulting injury to the urethra will depend upon the care of the operator and upon the presence or absence of a guide and upon the subsequent tearing in introducing the catheter for drainage. Most surgeons insert the catheter through the whole urethra, but the wound variation is considerable as to the extent of incision or laceration of the urethra and perineal tissues and the amount of subsequent packing or closure of these tissues. Some remove the catheter very early and some keep the wound open with repeated, extensive packing. If a guide has been passed, the only 
object of a perineal incision is to guard against infection; hence, all that is required is a small incision in the perineum down to the urethra, performed after the stricture has been cut with the Maisonneuve instrument and sounds passed to the desired size; if an external urethrotomy is done without a guide the perineal wound can be all but closed, and thus allow ample drainage. Later the wound could be opened easily if indicated. Hence, freshly incised tissues are approximated and heal quickly when held in apposition with mass sutures of silkworm gut, which should not be removed for at least a week. The catheter should remain this length of time also and repeatedly when removed there is little or no escape of urine through the nearly healed perineal wound in uncomplicated cases. The importance of leaving a minimum opening for drainage in clean cases and the proper retention of the catheter are essential for rapid and permanent healing. This is further demonstrated by the fact that success in plastic operations for cure of hypospadias depends upon wound closure and catheter retention until healed. Their tendency to fistula formation is well known by those who operate upon these obstinate cases. Prevention of recurrence of stricture will depend upon the faithfulness of the patient in attending to subsequent passing of sounds for an indefinite period.

So much for factors which tend to produce perineal fistulae and for measures for prevention of the same.

Method of Relieving These Cases. As already mentioned, the fistula persists for mechanical reasons, the urine following the path of least resistance. If there is resistance to the stream anywhere in the urethra distal to the opening which leads to the perineum, the fistula will tend to persist and the granulations fail to close it. Hence, all distal strictures must be relieved by full sound dilatation. Sometimes the introduction of a catheter for permanent drainage of bladder for a couple of weeks may avail. If these simple measures fail, some sort of perineal operation must be resorted to. These fistulae have often been opened down to the urethra and curetted, caustics applied, and other means resorted to, only to be followed by failure.

Technic. Each case may present some special feature, but the following general technic has never failed of speedy relief in the hands of the writer.

The operation should be deferred until all acute signs have subsided and only the fistulous tract remains. A guide should be passert through the whole urethra into the bladder. If there is no tight stricture, a moderate sized, steel sound (French 24) should be selected so as to distend the urethra at the fistulous opening; otherwise, a small guide should be used. Any stricture in the penile urethra should next be divided with the Otis or Maisonneuve instrument. Any narrowing at or near the fistulous opening, which is usually in the membranous urethra, should next be divided internally and preferably with the Maisonneuve instrument. Then a steel sound, French 24 , is passed and the patient put in the lithotomy position.

If no guide can be passed into the bladder, one should be introduced to the point of stricture, and the patient put in the above position. Next, the direction and length of the fistulous tract should be determined with a small probe. An oval incision is then made in the perineum. around the opening of the fistula, leaving a margin of skin, one-third of an inch wide, around it. If needed, a little more room may be gained by making an incision in the antero-posterior drrection at either end of the oval one, but this is not.

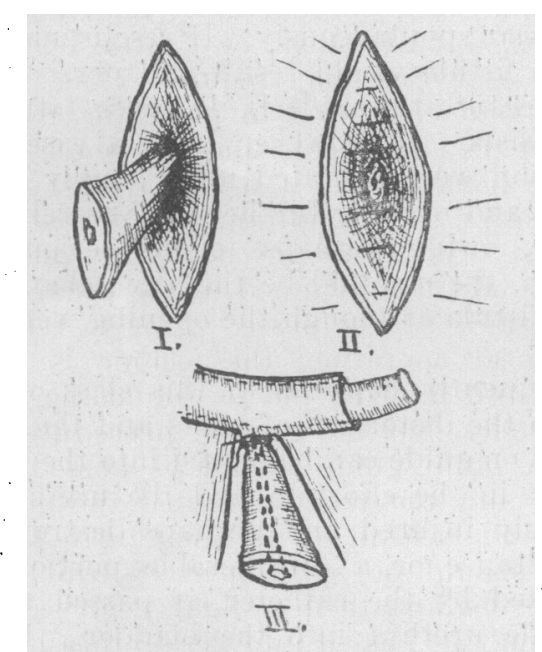

I. Fistulous tract dissected down to the urethra.

II. Tract excised and ligated at the urethra. Sutures placed.

II. Diagrammatic side-view showing tract dissected and ligated at the urethra which is distended with a steel sound.

often the case. The next step is the careful dissection of the tract so as to excise it without. opening it until the urethra is reached. Its wall should be kept reasonably thin, but should contain ample tissue so as not to break when the necessary traction is used. The dissecting will be chiefly through scar tissue. Frequent use of the probe will indicate the direction of the fistula or some sort of a guide may be left in the tract during the dissection. Stay sutures or some instrument should be used during the dissection for traction, and the dissection is nearly bloodless. The tract will vary from one to two inches in length. When the urethra has been reached a ligature of No. 1 chromicized catgut should be tied around the base of the tract, which is practically a tube, and the distal portion excised. Where it has been possible to insert a soft rubber catheter for permanent drainage prior to dissecting out the tract, this proves to be the best technic, because the catheter is as good a guide as the steel sound and, being already in position, there is no subsequent danger of injury after the parts have been dissected. Finally, the cut edges of the stump (tract) may 
be sutured with plain catgut. Then the walls of the incision are held in approximation with three or four interrupted, buried sutures of plain catgut. The edges of the skin, including :some of the deeper tissues, are sutured with silkworm gut or silver wire en masse so as to close the perineal wound. Owing to the fact that the dissection has been through scar tissue, there is but little chance for infection or infiltration. In thin subjects, where the fistulous tract is short, perineal drainage is not indicated but, as a precaution in certain cases, a very narrow strip of rubber dam may be inserted for twenty-four 'hours. The silkworm gut sutures should not be removed for about ten days. The after-treatment -does not differ from that of any patient wearing a catheter for bladder drainage. The perineal wound must be inspected daily and kept clean by lotions, wet dressings, or a little boric ointment, as each case may require. The catheter must be kept clean and in place for about ten .days. At the end of this time the wound should be about healed, but, if a few drops of urine :should escape, this condition will persist only a few days. As after any stricture operation, sounds should be continued in the usual way.

If no guide can be passed prior to beginning the perineal operation, the technic is a little more difficult in order not to wound the urethra unnecessarily, but the general procedure is the same. A blunt guide should be passed to the stricture and the tract dissected down to the urethra and, instead of its being ligated at this time, it should be split open its full length, when a probe and then a slightly curved, small director may be passed into the urethra in either direction. In the groove of the director a small Otis urethrotomy knife may be passed, dividing the stricture. After the passage of sounds the rubber catheter is to be inserted, the tract ligated and sutured and the wound closed as above described.

The appended cases are examples of the two conditions which may be met:

CASE I. This man, 38 years old, and in good general health had been operated upon successfully eight years before for stricture. In December, 1910, he entered the Boston City Hospital. Examination showed the presence of an impassable, deep stricture and a perineal fistula of about seven weeks' duration, through which all of his urine passed. He was operated upon by one of my colleagues who divided the stricture after enlarging the perineal wound and seeking the urethra with a guide. In so doing the urethra was opened for about two inches. The sinus was curetted and the wound packed after a catheter had been inserted through the whole urethra for bladder drainage. The catheter was removed in about seven days after which sounds were passed. It was one month later before any urine was passed through the penis. At the end of three months there remained a perineal fistula through which most of the urine escaped, although a French 28 sound could be passed.

The above was the condition when I saw this patient and the following operation was performed for the closure of the fistula. A small probe in the fistula came in contact with a steel sound previously passed, and the tract was about one inch long. With a Maisonneuve instrument several narrow places in the deep penile and membranous urethra were divided and then dilated to the size of a French 30 sound, and a soft rubber catheter, French 28, introduced with a stilette and left for permanent drainage. Patient was then put in the lithotomy position. Steps were carried out as given above in detail and briefly were as follows: Oval incision around the opening of the fistula leaving a narrow margin of skin a little enlargement of the wound antero-posteriorly, at either end of the oval incision, then a careful excision of the fistulous tract down to the urethra. The tract was then ligated with No. 1, chromicized catgut and excised, a strand of gut being left long to act as a drain. The wound was nearly closed with buried, plain catgut sutures and large mass sutures of silkworm gut for the skin and deeper tissues. Nothing but scar tissue was exposed by this dissection, which made a conical cavity narrowing at the urethra. The convalescence was uninterrupted. On the eighth day there was a slight leak of urine from the wound. Catheter. removed on the eleventh day and the stitches on the fourteenth day, at which time a few drops of urine escaped through a small perineal opening. A French 28 sound was passed on the seventeenth day and every five days thereafter. On the twenty-third day following operation the perineal opening was closed and remained permanently so and patient was passing a normal stream. Pathological examination of the excised tract showed only scar tissue.

CASE II. A man, 48 years old, operated upon six years ago for stricture. The perineal wound healed but one year later a local abscess formed and a discharging urinary fistula has since persisted. Examination shows a redundant scar in the center of which is a small opening leading to the urethra. $\mathrm{He}$ has also an impassable stricture and most of the urine passes through the fistula. He was operated upon April 12, 1912, at the Boston City Hospital. The technic differs from that used in the above case because no urethral guide could be passed. Briefly, it was as follows: A guide was passed down to a deep stricture. The fistulous tract was dissected out its full length and it was then split open anteriorly down to the urethra. A probe was passed into the bladder, then a small grooved director, on which an Otis urethrotomy knife was passed, thus cutting a moderate stricture proximal to the opening in the urethra. In a similar manner, a probe and then a director were passed into the penile urethra through the small fistulous opening, using care not to enlarge it, and the deep, penile stricture incised. A Maisonneuve instrument was easily passed and the knife used to ensure a full division of all strictures. The urethra was dilated with a French 30 sound and then a No. 28, soft rubber catheter introduced with a stilette. During all this manipulation great care was exercised not to injure the urethra unduly nor enlarge its fistulous opening. Finally, the base of the tract was ligated and excised, as in Case I, and the wound closed except for a strip of rubber tissue, one-eighth of an inch wide, which was removed on the second day. The catheter was removed on the fourteenth day. This wound healed by first intention, there being no escape of urine or exudate. It remained permanently closed and he passed a normal stream. 